\title{
Quality of life and efficacy of temozolomide combined with whole-brain radiotherapy in patients with brain metastases from non-small-cell lung cancer
}

\author{
YAJUAN LV, JIANDONG ZHANG, ZHEN LIU, NING LIANG and YUAN TIAN \\ Department of Radiology, Shandong Provincial Qianfoshan Hospital Affiliated \\ to Shandong University, Jinan, Shandong 250014, P.R. China
}

Received December 14, 2017; Accepted May 2, 2018

DOI: $10.3892 / \mathrm{mco} .2018 .1622$

\begin{abstract}
The aim of the present study was to investigate the efficacy and life quality of temozolomide (TMZ) combined with whole-brain radiotherapy (WBRT) for patients with brain metastases (BM) from non-small-cell lung cancer (NSCLC). A total of 77 cases of patients with lung cancer and BM were selected and divided into two groups; the first group was administered WBRT with TMZ, and the second group was administered WBRT with placebo. The efficacy, overall survival (OS) and quality of life (QoL) were then compared between the two groups. There were no significant differences in toxicity between the two groups. However, the first group exhibited an advantage over the second group in terms of objective response and OS $(\mathrm{P}<0.5)$. Therefore, WBRT may improve the QoL of patients with BM. TMZ concomitantly with WBRT was well-tolerated and may be recommended for the treatment of BM from NSCLC.
\end{abstract}

\section{Introduction}

Brain metastases (BM) are particularly frequent in lung cancer cases (40-50\%), and their presence considerably worsens the prognosis of the patients (1). It has been estimated that $25 \%$ of patients with non-small-cell lung cancer (NSCLC) also suffer from BM (2). Patients with BM often experience severe neurological symptoms and a poor quality of life (QoL). The overall survival (OS) of patients with BM from NSCLC is <3-6 months without treatment (3), and the 1-year survival rate is $<20 \%$ (4). Whole-brain radiotherapy (WBRT) may prolong survival, it is the standard of care for patients with $\mathrm{BM}$, and it is crucial for the control of distant microscopic

Correspondence to: Dr Jiandong Zhang, Department of Radiology, Shandong Provincial Qianfoshan Hospital Affiliated to Shandong University, 16766 Jingshi Road, Jinan, Shandong 250014, P.R. China E-mail: qianfoshan66@sina.cn

Key words: non-small-cell lung cancer, brain metastases, wholebrain radiotherapy, temozolomide, quality of life metastases (5). However, approximately half of patients with BM experience intracranial progression within a few months of RT (6). Chemotherapy has also exhibited limited efficacy in the treatment of BM due to the presence of the blood-brain barrier (7). The efficacy of tyrosine kinase inhibitors (TKIs), such as erlotinib or gefitinib, in central nervous system metastasis is modest, due to the limited cerebrospinal fluid penetration $(8,9)$; therefore, TKIs are generally used only for lung adenocarcinoma with epidermal growth factor receptor mutations (9). Temozolomide (TMZ) is able to cross the blood-brain barrier and has exhibited efficacy against a number of cancers with BM, including NSCLC (10). The efficacy of TMZ in patients with metastatic lung cancer has been previously demonstrated $(11,12)$. In addition to TMZ, certain alkylating agents, such as cisplatin, may exert an effect on BM by crossing the blood-brain barrier (5). TMZ with cisplatin has been confirmed to be an active and well-tolerated combination in patients with BM (13). The present study was designed to assess the efficacy and the associated QoL of WBRT combined with TMZ in patients with BM from NSCLC.

\section{Patients and methods}

Patients. Between January 2013 and July 2015, 77 cases of lung cancer patients with BM were selected and divided into two groups (details shown in Table I). The patients were required to meet the following criteria: i) First-time patients with pathologically diagnosed NSCLC and with no other prior tumors, and with a Karnofsky performance status (KPS) score $\geq 70$; ii) $\mathrm{BM}$ confirmed by magnetic resonance imaging (MRI) with at least 3 evaluations of cranial lesions and no previously administered cranial RT; iii) $18<$ age $<75$ years; iv) no other serious medical conditions, an expected survival $>3$ months and adequate renal, hepatic and hematological function. All the patients provided written informed consent, and the study protocol was approved by the Ethics Committee of the Qianfoshan Hospital Affiliated to Shandong University (Jinan, China).

Treatment. The first group of patients were administered WBRT (total dose of $30 \mathrm{~Gy} / 10$ fractions, $3 \mathrm{~Gy} /$ day on days $1-5$ weekly) and TMZ (75 mg/m² p.o. q.d., until the end of WBRT). The 
second group of patients were administered WBRT, at the same dose as the first group, with placebo (p.o. q.d. until the end of WBRT). Platinum-based chemotherapy was allowed in the two groups following WBRT, under the guidance of the doctors. The first group was administered TMZ $75 \mathrm{mg} / \mathrm{m}^{2}$ p.o. q.d. on days 1-5 combined with chemotherapy, and the second group was administered chemotherapy and placebo simultaneously. Mannitol and dexamethasone were given at the dose required in order to reduce the RT-related side effects. Treatment was continued until unacceptable toxicity occurred (such as grade 4 myelosuppression that did not improve after 1 week, or coma).

Response evaluation. Blood tests were performed once weekly, and a brain MRI was performed within 1 month of RT completion to evaluate the treatment efficacy. Following RT, all patients were evaluated every 2 months if they experienced no headaches or other symptoms. The evaluation included assessment of complications, physical examination and MRI scan of brain. The primary endpoints included objective response (OR), which was evaluated within 1 week after the end of WBRT. Response to treatment in the brain was assessed according to the World Health Organization/ Eastern Cooperative Oncology Group criteria, and defined as $\mathrm{CR}$ (complete remission), $\mathrm{PR}$ (partial remission), SD (stable disease) and PD (progressive disease). OR was defined as a measure of treatment efficacy $(\mathrm{CR}+\mathrm{PR})$. The secondary endpoints were overall survival (OS) and QoL. OS was defined as the time from RT initiation to death or the date of the last follow-up. QoL was measured using the Functional Assessment of Cancer Therapy-Brain (FACT-BR, version 4) before and after WBRT. Adverse reactions were evaluated according to the National Cancer Institute Common Terminology Criteria for Adverse Events, version 3.0 (14).

Statistical analysis. Data were analyzed using SPSS 22.0 software (IBM Corp., Armonk, NY, USA). General information on patients, such as OR and toxicity, were described and compared using the Chi-squared test. OS was calculated with the Kaplan-Meier method and the differences were compared using the log-rank test. The difference in QoL between the two groups prior to and following WBRT was compared by the independent samples t-test, and comparison of the QoL difference within each group before and after WBRT was performed using the paired samples t-test. $\mathrm{P}<0.05$ were considered to indicate a statistically significant difference.

\section{Results}

Patients. All the patients completed the treatment successfully. The patient characteristics are listed in Table I. The sample included a total of 49 male and 28 female patients, with a median age of 57 years and a median KPS score of 80 for the first group, and a median age of 61 years and a median KPS score of 80 for the second group. The sex ratios of male to female patients were 1.86 and 1.64 , respectively, in the two groups.

Treatment and efficacy. As shown in Table II, all the patients were followed up after the completion of RT. The first group was superior to the second group in terms of
Table I. General patient characteristics.

\begin{tabular}{lcc}
\hline Characteristics & Group 1 & Group 2 \\
\hline $\mathrm{N}$ & 40 & 37 \\
$\mathrm{Sex}$ & & \\
$\mathrm{Male}$ & 26 & 23 \\
Female & 14 & 14 \\
Median age, years & 57 & 61 \\
KPS score & & \\
$\leq 80$ & 15 & 10 \\
$>80$ & 25 & 27 \\
Histological type & & \\
Squamous cell carcinoma & 16 & 12 \\
Adenocarcinoma & 24 & 25 \\
Extracranial disease & & 17 \\
Absent & 22 & 20 \\
Present & 18 & \\
\hline
\end{tabular}

KPS, Karnofsky Performance Status.

OR $(\mathrm{P}<0.05)$. A total of 3 patients were lost to follow-up at 6, 8 and 12 months after RT. The median OS was 10 months [95\% confidence interval (CI): 8.0-12.0 months] for the first group and 8.5 months (95\% CI: 7.3-9.8 months) for the second group. The first group exhibited an advantage in terms of OS when compared with the second group ( $\mathrm{P}<0.05$; Fig. 1$)$. There was no significant difference between the two groups before and after RT (Table IV). However, QoL after RT was higher compared with that prior to RT in both groups (group 1, $\mathrm{P}<0.001$; group 2, $\mathrm{P}<0.001$; Fig. 2).

Safety. The toxicities observed are summarized in Table III. There was no significant difference in the side effects between the two groups. Hematological toxicity and vomiting in the first group were more severe compared with those in the second group, but the difference was not statistically significant. Only a few patients in both groups developed grade 3 or 4 toxicities.

\section{Discussion}

Lung cancer is the type of cancer that most commonly metastasizes to the brain. The median survival of patients with BM from lung cancer is $<3$ months (3). There are several treatment options for BM, such as WBRT, stereotactic body radiation therapy (SBRT), surgery and chemotherapy. The efficacy of SBRT and surgery is limited, as they may be applied to single small metastatic lesions, but BM generally manifest as multiple lesions. WBRT has been shown to increase the median survival in a number of trials, and it is considered as the standard treatment for BM $(15,16)$. The use of conventional chemotherapeutic drugs is limited, as it is difficult for these drugs to cross the blood-brain barrier. TMZ, which can penetrate through the blood-brain barrier, has demonstrated efficacy in BM, and may increase the effectiveness of WBRT (17). A meta-analysis including four trials demonstrated that the combination of TMZ and WBRT was superior to RT alone in 
Table II. Response of brain metastases after irradiation.

\begin{tabular}{lccrrrrrrr}
\hline Group & CR & PR & SD & PD & $\chi^{2}$ & P-value & OR & $\chi^{2}$ & P-value \\
\hline 1 & 8 & 19 & 8 & 5 & 5.43 & 0.14 & 27 & 4.59 \\
2 & 3 & 13 & 11 & 10 & & & 16 & 0.03 \\
\hline
\end{tabular}

CR, complete response; PR, partial response; $\mathrm{SD}$, stable disease; $\mathrm{PD}$, progressive disease; $\mathrm{OR}$, odds ratio.

terms of PR and SD. The combination group also exhibited a mild improvement compared with RT alone in terms of OS. However, the combination group also exhibited a significantly higher incidence of gastrointestinal and grade $>3$ hematological toxicities compared with the RT alone group (17). Another meta-analysis also demonstrated that concomitant administration of TMZ and RT significantly increased the OR in patients with BM compared with RT alone, but failed to demonstrate a survival advantage (18). A phase 2 trial demonstrated that the OR for TMZ combined with RT was significantly higher compared with that observed with $\mathrm{RT}$ alone $(\mathrm{P}=0.017)$. The median OS of the combination group was higher compared with the median OS of the RT alone group (8.6 vs. 7.0 months, respectively) (19). In another trial, the addition of TMZ to RT conferred an advantage in terms of PFS and OR $(\mathrm{P}<0.05)$, but there was no significant improvement in OS compared with RT alone. There was little difference in QoL between the two groups (20). A trial reported that the OR rate of concomitant WBRT with TMZ was $45 \%$. The median time to progression (TTP) was 9 months and the median OS was 13 months. The analysis of QoL in the trial revealed a high level of satisfaction among patients (21). Addition of TMZ to RT increased OR in patients with BM from NSCLC, as determined in a meta-analysis, but the differences in PFS and OS were not statistically significant (22). The efficacy and safety of TMZ were confirmed in these trials. However, other trials failed to demonstrate any significant improvements in OR or OS with the addition of TMZ to WBRT. A phase 3 trial reported that the combination of TMZ or erlotinib with RT did not improve OS in NSCLC patients with BM. The trial also demonstrated that the OS of erlotinib compared with that of TMZ in combination with RT did not differ significantly (23). Another phase 2 study reported no differences in TTP and OS between the $\mathrm{TMZ}+\mathrm{RT}$ and the RT alone groups (24), and a phase 3 trial reported that adding TMZ or erlotinib to RT conferred no OS benefit to patients with BM from NSCLC (8). In addition, the benefit of adding TMZ to WBRT has not been confirmed (11). Therefore, the administration of TMZ concomitantly with RT in patients with BM currently remains controversial.

In the present study, we reported an OR rate of $67.5 \%$ in the first group, while the OR rate was $43.2 \%$ in the second group. WBRT plus TMZ is a well-tolerated treatment with high response rates. In addition to its antitumor effect, TMZ may also increase the sensitivity of tumor cells to RT. The improvement in OS following the addition of TMZ to the treatment regimen may be due to the improved local control of BM. The Graded Prognostic Assessment (GPA) score may be used to evaluate the prognosis of lung cancer. An attempt was made to evaluate the effect of the GPA score on treatment

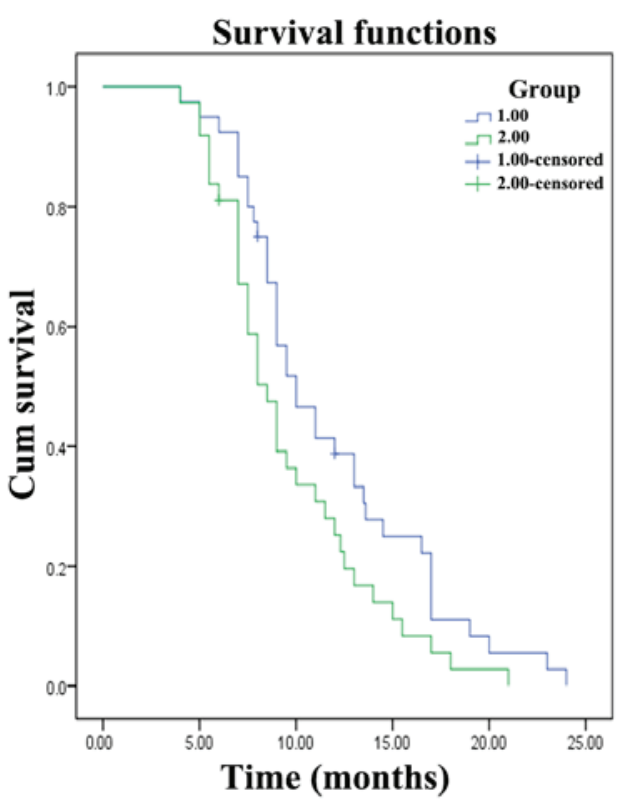

Figure 1. The OS of the two groups was calculated with the Kaplan-Meier method and the difference in OS between the two groups was compared using the log-rank test. Blue line, OS of the first group; green line, OS of the second group. OS, overall survival.

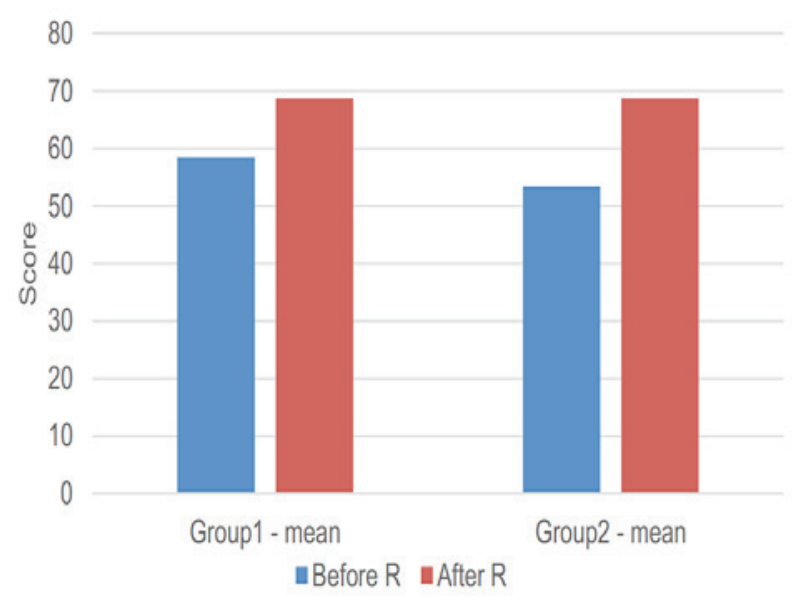

Figure 2. The difference in each group before and after radiotherapy.Mean score of QoL in the two group and comparison of the QoL difference in each group before and after WBRT was performed using the paired sample t test. The blue column indicates the average score before radiotherapy and the red column indicates the average score after radiotherapy. QoL, quality of life; WBRT, whole-brain radiotherapy.

efficacy and patient survival in the two groups, but there was no statistically significant difference. The reason may be that 
Table III. Treatment-related hematological and non-hematological toxicity.

\begin{tabular}{lcccccccc}
\hline & \multicolumn{2}{c}{ Group 1 } & & \multicolumn{2}{c}{ Group 2 } & & \\
\cline { 2 - 3 } Toxicity & Grade I-II & Grade III-IV & & Grade I-II & Grade III-IV & $\chi^{2}$ & & P-value \\
\hline Neutropenia & 19 & 10 & & 9 & 5 & 0.01 & 0.94 \\
Thrombocytopenia & 12 & 6 & & 5 & 1 & 0.60 & 0.63 \\
Anemia & 8 & 3 & & 5 & 2 & 0.00 & 1.00 \\
Vomiting & 15 & 9 & & 10 & 2 & 1.64 & 0.27 \\
Headache & 10 & 6 & & 12 & 3 & 1.15 & 0.43 \\
Vertigo & 13 & 5 & & 11 & 6 & 0.23 & 0.73 \\
\hline
\end{tabular}

Table IV. FACT-BR score before and after radiotherapy.

\begin{tabular}{lccccccr}
\hline & \multicolumn{3}{c}{ Before RT } & & \multicolumn{3}{c}{ After RT } \\
\cline { 2 - 3 } Group & BR score & $\mathrm{t}$ & P-value & & BR score & $\mathrm{t}$ & P-value \\
\hline 1 & $58.50(9.56)$ & 0.55 & 0.59 & & $68.78(7.74)$ & 0.51 & 0.61 \\
2 & $57.32(9.33)$ & & & $67.73(10.11)$ & & \\
\hline
\end{tabular}

FACT-BR, Functional Assessment of Cancer Therapy-Brain; RT, radiotherapy.

the sample size was limited. In addition, the lack of randomization is also a limitation of the present study, and the results including the value of GPA score require future confirmation by large-sample clinical trials in the future. Based on the results of this trial, TMZ is recommended for concomitant use with WBRT in patients with BM from lung cancer. PFS prolongation in patients with intracranial metastatic tumors is key to improving OS. Previous trials confirmed that TKIs combined with WBRT can prolong the OS when compared with WBRT alone, while other trials reported little benefit in terms of OS $(25,26)$. The reason for this inconsistency may be associated with the fact that the concentration of TKIs in the cerebrospinal fluid is markedly lower compared with their concentration in the serum. A large-sample trial is required to confirm the effect of TKIs on BM. The OS of patients with BM is shortened by treatment limitations, and the QoL of the patients is also becoming increasingly important. The QoL of patients with BM may be evaluated by FACT-BR, which has exhibited good reliability and validity (27). The QoL was not compromised in the TMZ combination group compared with the RT alone group in our trial, which is consistent with previous reports (28). The QoL of patients following RT was higher compared with that prior to RT, which may be due to the reduction of the tumor volume and relief of the peripheral nerve compression symptoms (28). Furthermore, psychological support and nursing interventions may improve QoL (29). The incidence of BM exhibits an increasing trend due to the increased incidence of cancer, but the OS of patients with BM was not significantly prolonged. Treatment of BM represents a major challenge, and there is an urgent need for novel drugs that are able to penetrate the blood-brain barrier.

\section{Acknowledgements}

Not applicable.

\section{Funding}

This study was supported by the National Natural Science Foundation of China (grant no. 81672974).

\section{Availability of data and materials}

The analysed data sets generated during the study are available from the corresponding author on reasonable request.

\section{Authors' contributions}

JZ designed this trial and was responsible for the final decision to submit for publication and had the full data of the paper. YL gathered data and wrote the report. ZL and NL helped to gather data. YT performed the literature search. All the authors have read and approved the final version of this manuscript.

\section{Ethics approval and consent to participate}

All procedures performed in studies involving human participants were in accordance with the ethical standards of the institutional and/or national research committee and with the 1964 Helsinki Declaration and its later amendments or comparable ethical standards. The study protocol was approved by the Ethics Committee of the Qianfoshan Hospital Affiliated to Shandong University, and all the patients provided written informed consent. 


\section{Consent for publication}

All the patients provided written informed consent.

\section{Competing interests}

The authors declare that they have no competing interests.

\section{References}

1. Seoane J and De Mattos-Arruda L: Brain metastasis: New opportunities to tackle therapeutic resistance. Mol Oncol 8: 1120-1131, 2014.

2. Berger LA, Riesenberg H, Bokemeyer C and Atanackovic D: CNS metastases in non-small-cell lung cancer: Current role of EGFR-TKI therapy and future perspectives. Lung Cancer 80 : 242-248, 2013

3. Sperduto PW, Kased N, Roberge D, Xu Z, Shanley R, Luo X, Sneed PK, Chao ST, Weil RJ, Suh J, et al: Summary report on the graded prognostic assessment: An accurate and facile diagnosis-specific tool to estimate survival for patients with brain metastases. J Clin Oncol 30: 419-425, 2012.

4. Tabouret E, Chinot O, Metellus P, Tallet A, Viens P and Gonçalves A: Recent trends in epidemiology of brain metastases: An overview. Anticancer Res 32: 4655-4662, 2012.

5. Baykara M, Kurt G, Buyukberber S, Demirci U, Ceviker N, Algin E, Coskun U, Aykol S,Emmez H,Ozet A, et al: Management of brain metastases from non-small cell lung cancer. J Cancer Res Ther 10: 915-921, 2014.

6. Scoccianti S and Ricardi U: Treatment of brain metastases: Review of phase III randomized controlled trials. Radiother Oncol 102: 168-179, 2012

7. Walbert $\mathrm{T}$ and Gilbert MR: The role of chemotherapy in the treatment of patients with brain metastases from solid tumors. Int J Clin Oncol 14: 299-306, 2009.

8. Sperduto PW, Wang M, Robins HI, Schell MC, Werner-Wasik M, Komaki R, Souhami L, Buyyounouski MK, Khuntia D, Demas W, et al: A phase 3 trial of whole brain radiation therapy and stereotactic radiosurgery alone versus WBRT and SRS with temozolomide or erlotinib for non-small cell lung cancer and 1 to 3 brain metastases: Radiation Therapy Oncology Group 0320. Int J Radiat Oncol Biol Phys 85: 1312-1318, 2013.

9. Chun SG, Choe KS, Iyengar P, Yordy JS and Timmerman RD: Isolated central nervous system progression on Crizotinib: An Achilles heel of non-small cell lung cancer with EML4-ALK translocation? Cancer Biol Ther 13: 1376-1383, 2012.

10. Tatar Z, Thivat E, Planchat E, Gimbergues P, Gadea E, Abrial C and Durando X: Temozolomide and unusual indications: Review of literature. Cancer Treat Rev 39: 125-135, 2013.

11. Chua D, Krzakowski M, Chouaid C, Pallotta MG, Martinez JI, Gottfried M, Curran W and Throuvalas N: Whole-brain radiation therapy plus concomitant temozolomide for the treatment of brain metastases from non-small-cell lung cancer: A randomized, open-label phase II study. Clin Lung Cancer 11: 176-181, 2010.

12. Gamboa-Vignolle C, Ferrari-Carballo T, Arrieta Ó and Mohar A: Whole-brain irradiation with concomitant daily fixed-dose temozolomide for brain metastases treatment: A randomised phase II trial. Radiother Oncol 102: 187-191, 2012.

13. Christodoulou C, Bafaloukos D, Linardou H, Aravantinos G, Bamias A, Carina M, Klouvas G and Skarlos D; Hellenic Cooperative Oncology Group: Temozolomide (TMZ) combined with cisplatin (CDDP) in patients with brain metastases from solid tumors: A Hellenic Cooperative Oncology Group (HeCOG) Phase II study. J Neurooncol 71: 61-65, 2005.

14. Trotti A, Colevas AD, Setser A, Rusch V, Jaques D, Budach V, Langer C, Murphy B, Cumberlin R and Coleman CN: CTCAE v3.0: Development of a comprehensive grading system for the adverse effects of cancer treatment. Semin Radiat Oncol 13: 176-181, 2003
15. Ferrarese F, Baggio V, Zorat PL and Fiore D: Treatment and prophylaxis for brain metastases from non-small cell lung cancer: whole brain radiation treatment versus stereotactic radiosurgery. Ann Oncol Suppl 2: ii71-ii72, 2006.

16. Larson D and Sahgal A; LarsonD and SahgalA: Adjuvant whole brain radiotherapy: strong emotions decide but rationale studies are needed: in regard to Brown et al. (Int J Radiat Oncol Biol Phys 2008;70:1305-1309). In reply to Drs. Larson and Sahgal. Int J Radiat Oncol Biol Phys 75: 316-317, 2009.

17. Liu R, Wang X, Ma B, Yang K, Zhang Q and Tian J: Concomitant or adjuvant temozolomide with whole-brain irradiation for brain metastases: A meta-analysis. Anticancer Drugs 21: 120-128, 2010.

18. Zhao Q, Qin Q, Sun J, Han D, Wang Z, Teng J and Li B: Brain radiotherapy plus concurrent temolomide versus radiotherapy alone for patients with brain metastases: A meta-analysis. PLoS One 11: e0150419, 2016

19. Antonadou D, Paraskevaidis M, Sarris G, Coliarakis N, Economou I, Karageorgis P and Throuvalas N: Phase II randomized trial of temozolomide and concurrent radiotherapy in patients with brain metastases. J Clin Oncol 20: 3644-3650, 2002.

20. Xia Deng, Zhen Zheng, Baochai Lin, et al:The efficacy and roles of combining temozolomide with whole brain radiotherapy in protection neurocognitive function and improvement quality of life of non-small-cell lung cancer patients with brain metastases. BMC cancer 17: 42, 2017.

21. Addeo R, Caraglia M, Faiola V, Capasso E, Vincenzi B, Montella L, Guarrasi R, Caserta L and Del Prete S: Concomitant treatment of brain metastasis with whole brain radiotherapy [WBRT] and temozolomide [TMZ] is active and improves quality of life. BMC Cancer 7: 18, 2007.

22. Ma W, Li N, An Y, Zhou C, Bo C and Zhang G: Effects of Temozolomide and Radiotherapy on Brain Metastatic Tumor: A Systematic Review and Meta-Analysis. World Neurosurg 92: 197-205, 2016.

23. Qin H, Pan F, Li J, Zhang X, Liang H and Ruan Z: Whole brain radiotherapy plus concurrent chemotherapy in non-small cell lung cancer patients with brain metastases: A meta-analysis. PLoS One 9: e111475, 2014.

24. Hassler MR, Pfeifer W, Knocke-Abulesz TH, Geissler K, Altorjai G, Dieckmann K and Marosi C: Temozolomide added to whole brain radiotherapy in patients with multiple brain metastases of non-small-cell lung cancer: A multicentric Austrian phase II study. Wien Klin Wochenschr 125: 481-486, 2013.

25. Zheng MH, Sun HT, Xu JG, Yang G, Huo LM, Zhang P, Tian JH and Yang $\mathrm{KH}$ : Combining Whole-Brain Radiotherapy with Gefitinib/Erlotinib for Brain Metastases from Non-Small-Cell Lung Cancer: A Meta-Analysis. BioMed Res Int 2016: 5807346 , 2016.

26. Broniscer A, Panetta JC, O'Shaughnessy M, Fraga C, Bai F, Krasin MJ, Gajjar A and Stewart CF: Plasma and cerebrospinal fluid pharmacokinetics of erlotinib and its active metabolite OSI-420. Clin Cancer Res 13: 1511-1515, 2007.

27. Chow R, Lao N, Popovic M, Chow E, Cella D, Beaumont J, Lam H, Pulenzas N, Bedard G, Wong E, et al: Comparison of the EORTC QLQ-BN20 and the FACT-Br quality of life questionnaires for patients with primary brain cancers: A literature review. Support Care Cancer 22: 2593-2598, 2014

28. Pulenzas N, Ray S, Zhang L, McDonald R, Cella D, Rowbottom L, Sahgal A, Soliman H, Tsao M, Danjoux C, et al: The Brain Symptom and Impact Questionnaire in brain metastases patients: A prospective long-term follow-up study. CNS Oncol 5: 31-40, 2016.

29. Pan CJ, Liu HC, Liang SY, Liu CY, Wu WW and Cheng SF: Resilience and coping strategies influencing the quality of life in patients with brain tumor. Clin Nurs Res Jun 1: 1054773817714562,2017 